\title{
Cross Ambiguity Function Based Integer Frequency Offset Estimation for OFDM Systems
}

\author{
Danping Li, Yongzhao Li, and Hailin Zhang \\ State Key Laboratory of Integrated Services Networks \\ Xidian University, Xian, 710071, China \\ Email: dpli@mail.xidian.edu.cn, \{yzhli, hlzhang\}@xidian.edu.cn
}

\author{
Lei Wang \\ School of Electronic Engineering \\ Xidian University, Xian, 710071, China \\ Email: leiwang@mail.xidian.edu.cn
}

\begin{abstract}
Using only one training symbol, a novel cross ambiguity function (CAF) based integer frequency offset (IFO) estimator for OFDM systems is proposed. From the energy distribution characteristics of the ideal CAF, an energy-detection based metric is obtained. By designing a training symbol whose ambiguity function (AF) is a valid approximation of the ideal thumbtacktype AF, a high-accuracy and full-range IFO estimation can be achieved over frequency-selective fading channels. Furthermore, the adoption of the CAF expression in terms of time-domain signals keeps the complexity of the proposed algorithm at a relatively low level. Simulation results verify its superior accuracy of the IFO estimation over conventional algorithms.
\end{abstract}

\section{INTRODUCTION}

Orthogonal frequency division multiplexing (OFDM) has been shown to possess high spectral efficiency and robustness against frequency-selective fading [1]. However, a well-known problem of OFDM is its susceptibility to the frequency offset that is mainly caused by Doppler shifts and/or local oscillators mismatch between the receiver and the transmitter [2]. In practice, the normalized frequency offset can usually be divided into an integer part (multiple of the subcarrier spacing) and a fractional part (less than one-half of the subcarrier spacing) so that they can be estimated separately. If not accurately estimated and compensated, the fractional frequency offset can destroy the orthogonality of the subcarriers and result in inter-carrier interference, while the integer frequency offset (IFO) will lead to a circular shift of the subcarrier indices and degrade performance.

Currently, commonly-used IFO estimation approaches are training assisted and implemented via time-domain or frequency-domain correlations [3]-[6]. A repetitive-structure training symbol is proposed in [3], and the frequency offset is estimated via time-domain correlation. However, the estimation range is limited by the number of identical parts, and the number of identical subparts. To obtain a full acquisition range, frequency-domain correlation based approaches are usually adopted. In the algorithm proposed by Schmidl and Cox, which we label S\&C [4], two consecutive differentially encoded training symbols in frequency domain are utilized. An improvement to the $\mathrm{S} \& \mathrm{C}$ approach, which is referred to as ML IFO estimation, is proposed in [5] in which the IFO estimation is also carried out by frequency-domain correlations. Two training symbols are required in the aforementioned algorithms, resulting in a lower efficiency in bandwidth. To reduce the training overhead, a differential OFDM (DOFDM) based IFO estimation method using only one training symbol is proposed in [6]. However, the estimation performance would degrade severely in the presence of frequency-selective fading channels.

In this paper, by using only one training symbol, a high-accuracy IFO estimation algorithm with robustness to frequency-selective fading is proposed. More specifically, an energy-detection based estimation metric is constructed according to the energy distribution of the cross ambiguity function (CAF). The superior estimation resolution can be achieved as long as the training symbol has an ambiguity function (AF) surface with only one single sharp peak, which is a valid approximation of the ideal thumbtack-type $\mathrm{AF}$ surface and can be easily designed in the frequency domain. Furthermore, the computational complexity of the proposed algorithm can be kept at a relatively low level by utilizing the CAF expression in terms of time-domain signals.

Notations: The superscript ${ }^{T}$ and ${ }^{*}$ denote transpose and complex conjugate, respectively; $\|\cdot\|$ denotes Euclidean norm; $\mathbf{C}(m,:)$ and $\mathbf{C}(:, n)$ denote the $m^{\text {th }}$ row and $n^{\text {th }}$ column of matrix $\mathbf{C}$, respectively; $\langle\cdot\rangle_{N}$ represents the modulo- $N$ operation and $\delta(\cdot)$ is the Dirac delta function; $\mathcal{N}_{\mathcal{C}}\left(\mu, \sigma^{2}\right)$ is a complex normal distribution with mean $\mu$ and variance $\sigma^{2}$; and $\chi^{2}(k)$ is a chi-square distribution with $k$ degrees of freedom.

\section{SySTEM MODEL}

Consider a discrete-time baseband OFDM system with the lengths of the IFFT/FFT and cyclic prefix (CP) equal to $N$ and $N_{g}$, respectively. We assume $x(n)(0 \leq n \leq N-1)$ is the local training symbol for IFO estimation, which is generated as follows:

$$
x(n)=\frac{1}{N} \sum_{k=0}^{N-1} X(k) e^{j 2 \pi k n / N}
$$

where $X(k)(0 \leq k \leq N-1)$ is the corresponding frequency-domain training symbol. Assuming that the length of the discrete-time channel impulse response (CIR) is $L$, we denote $\mathbf{h}=[h(0), h(1), \cdots, h(L-1)]^{T}$ and $H(k)=$ $\sum_{l=0}^{L-1} h(l) e^{-j 2 \pi l k / N}$ as the discrete-time CIR and the channel frequency response, respectively. Moreover, the channel is assumed to be time invariant over the OFDM block duration. After being transmitted through the channel and corrupted by 
Gaussian noise, the received training block can be expressed as

$$
y(n)=\sum_{l=0}^{L-1} h(l) x\left(\langle n-l\rangle_{N}\right) e^{j\left(2 \pi \varepsilon n / N+\theta_{0}\right)}+w(n)
$$

where $w(n)$ is the time-domain complex AWGN; $\varepsilon$ and $\theta_{0}$ are the normalized IFO and initial phase, respectively. Without loss of generality, we assume $\theta_{0}=0$ for simplicity. Since IFO is the focus here, we assume that the fractional frequency offset and timing offset have been perfectly estimated and compensated. Then, the FFT output of the received block is

$$
Y(k)=H\left(\langle k-\varepsilon\rangle_{N}\right) X\left(\langle k-\varepsilon\rangle_{N}\right)+W(k)
$$

where $W(k)$ represents the frequency-domain complex AWGN with zero mean and variance $\sigma_{\mathrm{n}}^{2}$.

\section{CAF BASED IFO ESTIMATION}

\section{A. Energy-detection Based IFO Estimation metric}

AFs were initially applied in target resolution and parameter estimation for radar systems [7]-[8]. Applications of AF can also be found in pulse design and optimization for OFDM systems [9]-[10].

To have a better understanding of the proposed IFO algorithm, we review the concepts of $\mathrm{AF}$ and $\mathrm{CAF}$ as follows. $\mathrm{AF}$, a 2-D function of time delay and frequency offset, is defined as the inner product of a signal and its time-delayed, frequencyshifted version [7]. The AF of the local training symbol $x(n)$, denoted as $\mathbf{A}_{\mathbf{x x}}$, is given by

$$
A_{\mathbf{x x}}(\tau, v)=\sum_{n=0}^{N-1} x(n) x^{*}\left(\langle n-\tau\rangle_{N}\right) e^{-\frac{j 2 \pi v n}{N}}
$$

where $\tau$ and $v$ represent the time delay and frequency offset, respectively. As is well known, it is more convenient for OFDM systems to design the training symbol in the frequency domain. Hence, the AF $\mathbf{A}_{\mathbf{x x}}$ can be alternatively expressed by the corresponding frequency-domain signal $X(k)$, i.e.,

$$
A_{\mathbf{x x}}(\tau, v)=\frac{1}{N} \sum_{k=0}^{N-1} X(k+v) X^{*}(k) e^{\frac{j 2 \pi k \tau}{N}}
$$

Similarly, the CAF, which is denoted as $\mathbf{A}_{\mathbf{y x}}$, of the received training block $y(n)$ and the local training symbol $x(n)$ can be computed using the corresponding frequency-domain signals as follows

$$
A_{\mathbf{y x}}(\tau, v)=\frac{1}{N} \sum_{k=0}^{N-1} Y(k+v) X^{*}(k) \cdot e^{\frac{j 2 \pi k \tau}{N}}
$$

Moreover, we define the following variables, as

- $\left|\mathbf{A}_{\mathbf{x x}}\right|^{2}$ : AF surface, the set of $\left\{\left|A_{\mathbf{x x}}(\tau, v)\right|^{2}\right\}$

- $\left|\mathbf{A}_{\mathbf{y x}}\right|^{2}$ : CAF surface, the set of $\left\{\left|A_{\mathbf{y x}}(\tau, v)\right|^{2}\right\}$

- $\boldsymbol{\Gamma}_{v}=\mathbf{A}_{\mathbf{x x}}(:, v)$ : the $v^{\text {th }}$ AF Doppler cut

- $\boldsymbol{\Psi}_{v}=\mathbf{A}_{\mathbf{y x}}(:, v)$ : the $v^{\text {th }}$ CAF Doppler cut

Using (6) and (3), the relationship between $\boldsymbol{\Psi}_{v}$ and $\boldsymbol{\Gamma}_{v}$ can be written as

$$
\Psi_{v}(\tau)=\sum_{m=0}^{L-1} e^{-\frac{j 2 \pi m(v-\varepsilon)}{N}} h(m) \Gamma_{v-\varepsilon}\left(\langle\tau-m\rangle_{N}\right)+G_{v}(\tau)
$$

where $G_{v}(\tau) \triangleq \frac{1}{N} \sum_{k=0}^{N-1} W(k+v) X^{*}(k) e^{\frac{j 2 \pi k \tau}{N}}$ is the noise term. This shows that a CAF Doppler cut is a linear transformation of an AF Doppler cut corrupted by additive noise $G_{v}(\tau)$.

It is well known that an AF surface is ideal if it is nonzero only at $(0,0)$ on the 2-D time-delay/frequency-offset plane [11]. Obviously, the corresponding AF can be expressed as

$$
A_{\mathbf{x x}}(\tau, v)=\left\{\begin{array}{cc}
Q & \tau=v=0 \\
0 & \text { otherwise }
\end{array}\right.
$$

where $Q$ is a real positive constant. This type of $\mathrm{AF}$ is usually referred to as an ideal thumbtack-type AF, which could yield superior target-resolution capabilities [11]. Then, from (7) and (8), the corresponding ideal $\mathrm{CAF}^{*}$ Doppler cuts have the following expression

$$
\Psi_{v}(\tau)=\left\{\begin{array}{cc}
Q \cdot h(\tau)+G_{v}(\tau) & v=\varepsilon, 0 \leq \tau \leq L-1 \\
G_{v}(\tau) & \text { otherwise }
\end{array}\right.
$$

From (9) and (7), we can show that, on the 2-D timedelay/frequency-offset plane, $A_{\mathbf{y x}}(\tau, v)$ and $h(\tau)$ are correlated only in the region $\{(\tau, v) \mid 0 \leq \tau \leq L-1 ; v=\varepsilon\}$. Base on this observation, if $\mathbf{h}$ is available for the IFO estimation, an intuitive method is to compute the cross-correlations of $\mathbf{h}$, and every Doppler cut $\Psi_{v}$ in the region $\Omega=\{(\tau, v) \mid 0 \leq \tau \leq$ $\left.L-1,-\varepsilon_{\max } \leq v<\varepsilon_{\max }\right\}$; then an estimate of IFO can be obtained by determining the maximum modulus. Denote $\hat{\varepsilon}$ as the trail values of IFO, then the aforementioned metric for IFO estimation based on the peak-detection of the crosscorrelations can be constructed as

$$
\hat{\varepsilon}=\underset{-\varepsilon_{\max } \leq \hat{\varepsilon}<\varepsilon_{\max }}{\arg \max }\left|\sum_{\tau=0}^{L-1} h(\tau)^{*} \Psi_{\hat{\varepsilon}}(\tau)\right|
$$

Unfortunately, (10) is hard to be realized since a valid channel estimation could not be obtained before frequency offset is estimated and compensated. Nevertheless, from (9), an estimate of CIR, $\hat{\mathbf{h}}$, can be constructed as

$$
\hat{h}(\tau)=\Psi_{\hat{\varepsilon}}(\tau) / Q
$$

where $0 \leq \tau \leq L-1$. This means that, for a given $\hat{\varepsilon}$, a corresponding channel estimation $\hat{\mathbf{h}}$ can be obtained. Substituting (11) into (10), IFO estimation based on an ideal energy distribution $\hat{\varepsilon}$ can be obtained by finding $\hat{\varepsilon}$ to maximize the following metric

$$
M(\hat{\varepsilon})=\sum_{\tau=0}^{L-1}\left|\Psi_{\hat{\varepsilon}}(\tau)\right|^{2}
$$

\section{B. Training Symbol Design}

Although the ideal thumbtack-type AF can provide an ideal frequency offset resolution, unfortunately, such a shape of AF is impossible to attain [12]. Naturally, next step is to find a practical training symbol whose $\mathrm{AF}$ can approximate the ideal thumbtack-type AF. In turn, IFO estimation with high

\footnotetext{
*Unless otherwise stated, "CAF of the training symbol" in this paper represents the $\mathrm{CAF}$ of the received training block and the local training symbol.
} 


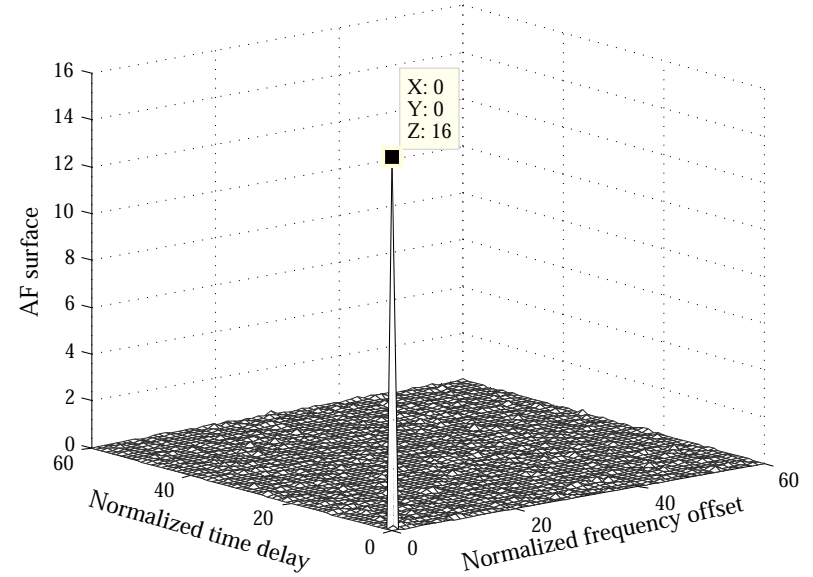

Fig. 1. AF surface $(N=512, \alpha=2)$

accuracy can be obtained by adopting the same metric in (12). By investigating several commonly used training symbols for OFDM systems, a frequency-domain symbol (denoted by $X(k)$ ) with constant amplitude $(\alpha)$ and random phase is chosen. Usually, $X(k)$ can be approximately modeled as i.i.d. random variables (RV) with zero mean and variance $\alpha^{2}$.

In order to verify the validity of the selected training symbol, the AF characteristics for $X(k)$ are analyzed and compared with the ideal thumbtack-type AF from the perspective of energy distribution. For any given $v(0 \leq v \leq N-1)$, from (5), the energy of the $v^{\text {th }}$ AF Doppler cut is given by

$$
\left\|\boldsymbol{\Gamma}_{v}\right\|^{2}=\alpha^{4}
$$

which implies that all AF Doppler cuts have the same energy. Using (5), it is easy to show that the $0^{\text {th }}$ (i.e., $v=0$ ) $\mathrm{AF}$ Doppler cut is

$$
\Gamma_{v}(\tau)=\alpha^{2} \delta(\tau)
$$

Then, it is obvious that the energy of the $0^{\text {th }}$ AF Doppler cut is concentrated at the $0^{\text {th }}$ time delay.

For $v \neq 0$, using the statistical characteristics of $X(k)$, the Central Limit Theorem and (5), we can deduce that $\left\{\Gamma_{v}(\tau)\right\}$ are nearly identically distributed complex Gaussian RVs with zero mean and variance $\sigma_{\gamma}^{2}=\alpha^{4} / N$, i.e.,

$$
\Gamma_{v}(\tau) \sim \mathcal{N}_{\mathcal{C}}\left(0, \alpha^{4} / N\right), \text { for } v \neq 0
$$

In turn, $\left\{\left|\Gamma_{v}(\tau)\right|^{2}\right\}$ are nearly identically distributed complex $\chi_{2}^{2}$-distributed RVs with degree of freedom 2, i.e., $\left|\Gamma_{v}(\tau)\right|^{2} \sim$ $\frac{\sigma_{\gamma}^{2}}{2} \chi^{2}(2)$. This conclusion implies that each element of the $v^{\text {th }}(v \neq 0)$ AF Doppler cut has an average energy of $\alpha^{4} / N$. Hence, the total energy $\alpha^{4}$ of the $v^{\text {th }}(v \neq 0)$ AF Doppler cut is uniformly spread over all $N$ time delays in the statistical sense.

From the above analyses of the AF energy distribution, we can see that the proposed training symbol has only one single sharp peak on its AF surface as shown in Fig. 1. This verifies that the $\mathrm{AF}$ of the proposed training symbol is a valid approximation of the ideal thumbtack-type AF.

The CAF characteristics of the proposed training symbol can be analyzed in a similar way as follows. Since $\{W(k)\}$ are i.i.d. Gaussian RVs with the distribution of $\mathcal{N}_{\mathcal{C}}\left(0, \sigma_{\mathrm{n}}^{2}\right)$ and

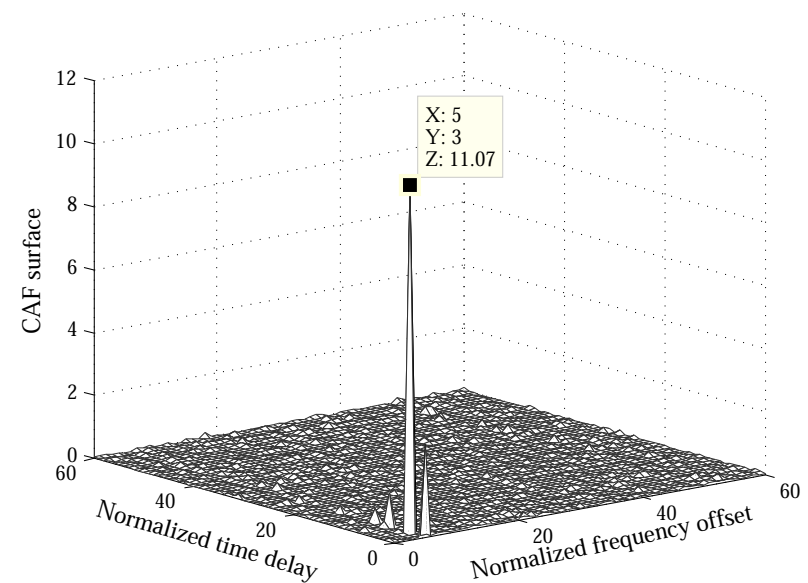

Fig. 2. CAF surface $(N=512, \alpha=2, \varepsilon=5, L=26, \eta=25 \mathrm{~dB}$, $\left.\max \left\{|h(n)|^{2}\right\}=0.6919\right)$

are independent of $\{X(k)\}$, we can show that the noise term $G_{v}(\tau)$ in (7) are identically distributed zero-mean complex Gaussian RVs with variance $\sigma_{\mathrm{g}}^{2}$. Since $\{X(k)\}$ have a constant amplitude $\alpha, \sigma_{\mathrm{g}}^{2}$ can be given by $\sigma_{\mathrm{g}}^{2}=\alpha^{2} \sigma_{\mathrm{n}}^{2} / N$. Then, using (7) and (13), the energy of the $v^{\text {th }}(0 \leq v \leq N-1)$ CAF Doppler cut can be computed as

$$
\begin{aligned}
\left\|\boldsymbol{\Psi}_{v}\right\|^{2} & =\sum_{\tau=0}^{N-1}\left|\Psi_{v}(\tau)\right|^{2} \approx\left\|\boldsymbol{\Gamma}_{v}\right\|^{2} \sum_{l=0}^{L-1}|h(l)|^{2}+N \sigma_{\mathrm{g}}^{2} \\
& =\alpha^{4} \sum_{l=0}^{L-1}|h(l)|^{2}\left(1+\eta^{-1}\right)
\end{aligned}
$$

where $\eta \triangleq \frac{\alpha^{2}}{\sigma_{\mathrm{n}}^{2}} \sum_{l=0}^{L-1}|h(l)|^{2}$ is the signal-to-noise ratio (SNR). From (16) we can see that, all CAF Doppler cuts have approximately the same energy. It also should be noted that, the approximation in (16) will approach the equality with an increase in $N$ and/or $\eta$.

Then, we investigate the energy distribution of the CAF Doppler cut whose index $v$ equals the true IFO $\varepsilon$. Using (7) and (14), we get

$$
\Psi_{v}(\tau)=\alpha^{2} h(\tau)+G_{v}(\tau), \text { for } 0 \leq \tau \leq L-1
$$

Since $h(\tau)$ is defined over [0,L-1], then we have

$$
\begin{aligned}
& \sum_{\tau=0}^{L-1}\left|\Psi_{v}(\tau)\right|^{2} \approx \alpha^{4} \sum_{l=0}^{L-1}|h(l)|^{2}+L \sigma_{\mathrm{g}}^{2} \\
& =\alpha^{4} \sum_{l=0}^{L-1}|h(l)|^{2}\left(1+\frac{L}{N} \eta^{-1}\right)
\end{aligned}
$$

Similar to (16), the approximation in (18) will approach the equality with an increase in $N$ and/or $\eta$.

On the other hand, for $v \neq \varepsilon$, we can show that $\left\{\left|\Psi_{v}(\tau)\right|^{2}\right\}$ are nearly identically distributed complex $\chi^{2}$-distributed RVs with degree of freedom 2 , i.e.,

$$
\left|\Psi_{v}(\tau)\right|^{2} \sim \frac{\sigma_{\psi}^{2}}{2} \chi^{2}(2)
$$

where $\sigma_{\psi}^{2}=\sigma_{\mathrm{g}}^{2}+\sigma_{\gamma}^{2} \sum_{l=0}^{L-1}|h(l)|^{2}=\frac{\alpha^{4}}{N} \sum_{l=0}^{L-1}|h(l)|^{2}(1+$ $\left.\eta^{-1}\right)$. Similar to AF which has a uniformly spread energy when $v \neq 0$, the energy of the $v^{\text {th }}(v \neq \varepsilon)$ CAF Doppler 
cut is also uniformly spread over all $N$ time delays in the statistical sense.

Therefore, we conclude that for a frequency-domain training symbol with constant amplitude and random phase, the main sharp peaks only appear over the first L time delays on the CAF Doppler cut when $v=\varepsilon$ (see Fig. 2). This demonstrates the validity of the proposed training symbol, which ensures that the metric in (12) is applicable for the proposed training sequence to achieve high-accuracy IFO estimation.

\section{Performance Analysis}

Using the metric in (12), a CAF based IFO estimator is constructed as follows,

$$
\hat{\varepsilon}=\underset{-\varepsilon_{\max } \leq \hat{\varepsilon}<\varepsilon_{\max }}{\arg \max } M(\hat{\varepsilon})=\underset{-\varepsilon_{\max } \leq \hat{\varepsilon}<\varepsilon_{\max }}{\arg \max } \sum_{\tau=0}^{L-1}\left|\Psi_{\hat{\varepsilon}}(\tau)\right|^{2}
$$

Since $M(\hat{\varepsilon})$ is just the energy of the $\hat{\varepsilon}^{\text {th }}$ CAF Doppler cut over $[0, L-1]$, we analyze the performance from the perspective of the CAF energy distribution. From Sec. III-B, we can deduce that, at high SNR region, the maximum value of the energy in (20) will be obtained when $\hat{\varepsilon}=\varepsilon$. Moreover, since the domain of definition of $v$ in CAF is $[-N / 2, N / 2)$, the effective searching range of $\hat{\varepsilon}$ can be up to $[-N / 2, N / 2)$. This means that the proposed algorithm has the capability of fullrange IFO estimation, the same as for DOFDM, S\&C and ML.

Moreover, probabilities of correct and incorrect estimations are analyzed. First, as described in Sec. III-B, for the proposed training symbol, an increase in $N$ will result in a sharper main peak around $(0,0)$ on its AF surface. Then, for a given SNR, a larger energy difference between the $v^{\text {th }}(v=\varepsilon)$ CAF Doppler cut and any other CAF Doppler cut $(v \neq \varepsilon)$ in the region $\Omega=$ $\left\{(\tau, v) \mid 0 \leq \tau \leq L-1,-\varepsilon_{\max } \leq v<\varepsilon_{\max }\right\}$ is consequently provided. On the other hand, it is obviously that an increase in $\eta$ will make the energy difference more distinct. Hence, the accuracy of IFO estimation will be improved with an increase in $N$ and/or $\eta$. The impact of $N$ and $\eta$ on the IFO estimation can be alternatively explained from the probability of incorrect IFO estimations. From (19) we can show that, for an incorrect $\hat{\varepsilon}, M(\hat{\varepsilon})$ has a distribution of $\frac{\sigma_{\psi}^{2}}{2} \chi^{2}(L)$ with mean $L \sigma_{\psi}^{2}$ and variance $L \sigma_{\psi}^{4}$, where $\sigma_{\psi}^{2}=\frac{1}{N}\left(\alpha^{2} \sigma_{n}^{2}+\alpha^{4} \sum_{l=0}^{L-1}|h(l)|^{2}\right)=$ $\frac{\alpha^{4}}{N} \sum_{l=0}^{L-1}|h(l)|^{2}\left(1+\eta^{-1}\right)$. Hence, for a given CIR length $L$, an increase in $N$ and/or $\eta$ will result in a decrease in both the expected value and variance of $M(\hat{\varepsilon})$. In other words, the probability of incorrect IFO estimation is reduced.

Moreover, the complexity of the proposed IFO estimation scheme is analyzed. From (20) we can see that, in order to obtain a full acquisition range IFO estimation, $N$ Doppler cuts of the CAF are involved. As stated earlier, the Doppler cuts of CAF can be computed using either frequency-domain or time-domain signals. When the frequency-domain signals based expression (6) is used, $N$ IFFT operations are required. However, we can alternatively represent the CAF in terms of

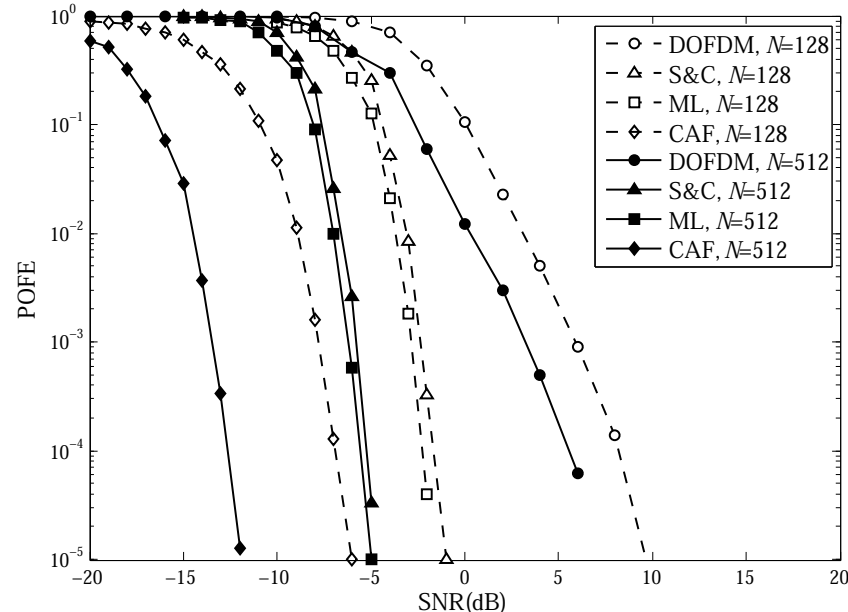

Fig. 3. Performance comparison for AWGN channels

time-domain signals, as

$$
A_{\mathbf{y x}}(\tau, v)=\sum_{n=0}^{N-1} y(n) x^{*}\left(\langle n-\tau\rangle_{N}\right) e^{-\frac{j 2 \pi v n}{N}}
$$

where $\tau \in[0, L-1], v \in[-N / 2, N / 2)$ represent the time delay and frequency offset, respectively. Obviously, in (21), only $L$ FFT operations are needed, while $L$ is usually far less than $N$ for OFDM systems. By substituting (21) into (20), a lower complexity IFO estimation is given by

$$
\hat{\varepsilon}=\underset{-N / 2 \leq \hat{\varepsilon}<N / 2}{\arg \max } \sum_{\tau=0}^{L-1}\left|\sum_{n=0}^{N-1} y(n) x^{*}\left(\langle n-\tau\rangle_{N}\right) e^{-\frac{j 2 \pi \hat{\varepsilon} n}{N}}\right|^{2}
$$

It should be noted that, although (21) has a different expression from (6), the estimation performance will be unchanged since they have absolutely identical values.

\section{Simulation Results}

The validity of the proposed CAF based algorithm is verified in terms of the probability of failure estimation (POFE) defined as $\operatorname{Pr}\{\hat{\varepsilon} \neq \varepsilon\}$. Two typical IEEE 802.11e OFDMA parametersets, $\left(N=128 ; N_{g}=16\right)$ and $\left(N=512 ; N_{g}=64\right)$, are adopted. A full acquisition range $[-N / 2, N / 2)$ is considered. For each SNR, at least $10^{6}$ independent trials are performed in which the IFOs are randomly generated within $[-N / 2, N / 2)$. In particular, the POFEs of the DOFDM, S\&C and ML are simulated for comparison, where S\&C and ML require two consecutive OFDM training symbol, and the others require only one OFDM training symbol.

From POFEs for AWGN channels as shown in Fig. 3, we can see that the proposed algorithm outperforms the conventional DOFDM, S\&C and ML approaches. Moreover, the performance improvement is more obvious for the CAF based algorithm than the other approaches when the length of OFDM training symbol increases.

Fig. 4 compares the performance of different algorithms for frequency-selective fading channels. Without loss of generality, COST207 RA model [1] with $L=6$ is adopted. Similar to AWGN channels, the CAF IFO estimation also 


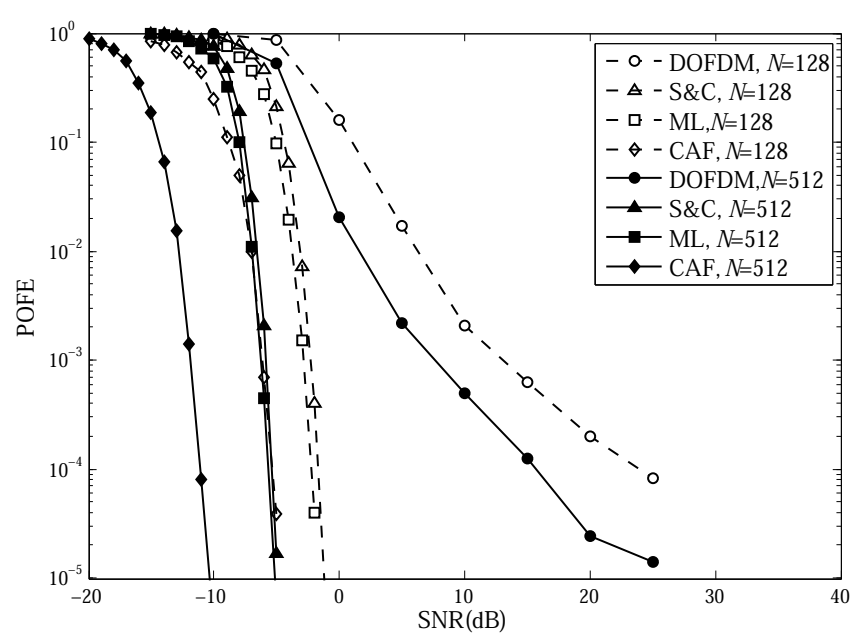

Fig. 4. Performance comparison for COST207 RA channels

outperforms DOFDM, S\&C and ML algorithms for frequencyselective fading channels. Moreover, as shown in Figs. 34, S\&C and ML almost have the same performance for both AWGN and frequency-selective fading channels, while DOFDM is very sensitive to the frequency selectivity of the channel. As a one training symbol based algorithm, CAF based IFO estimation degrades slightly in frequency-selective fading channels compared with AWGN channels. However, it still outperforms the S\&C and ML with a lower training overhead. Another observation from Figs. 3-4 is an increase in the length of FFT $(N)$ results in a performance improvement for all candidates. The reason for the performance improvement of CAF based IFO estimation can be found in Sec. III-C.

Further, Fig. 5 shows the POFEs of CAF IFO estimation for different frequency-selective fading channel models. Three 6path channel models with different lengths of CIR are adopted: COST207 TU $(L=51)$, ITU VA $(L=26)$ and COST207 RA $(L=6)$. It is observed that a decrease in the sparsity of frequency-selective fading channel improves the performance of the CAF based algorithm. The reason is, in sparse channels, the accuracy of IFO is reduced since more taps that only contain noise term are involved in IFO metric. Hence, if the knowledge of channel length and position of channel taps are available, they can be utilized to improve the performance of the IFO estimation; this will be considered as future work.

\section{CONCLUSiONS}

In this paper, we focus on the IFO estimation in OFDM systems over frequency-selective fading channels using only one training symbol. By analyzing the energy distribution of the CAF, an energy-detection based estimation metric is constructed. Specifically, an OFDM symbol with constant amplitude and random phase in frequency domain is adopted as the training symbol. Its $\mathrm{AF}$ and $\mathrm{CAF}$ are verified to be valid approximations to the ideal $\mathrm{AF}$ and $\mathrm{CAF}$, respectively. As a result, a high-accuracy and full-range IFO estimator is obtained in the presence of frequency-selective fading. Furthermore, the computational complexity of the proposed CAF based IFO estimator can be kept at a relatively low

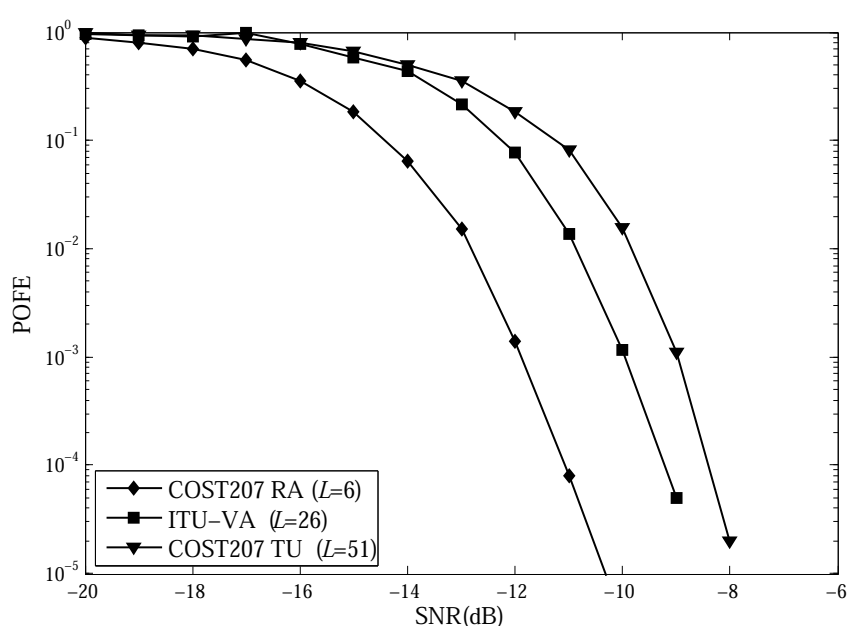

Fig. 5. Performance comparison for different frequency-selective fading channels $(N=512)$

level by utilizing the CAF expression in terms of time-domain signals.

\section{ACKNOWLEDGMENT}

This work was supported by the National Natural Science Foundation of China (No.61072069), the Important National Science \& Technology Specific Projects (2011ZX03003-00104, 2012ZX03003012-003), the Fundamental Research Funds for the Central Universities (No.72001859), the State Key Laboratory of Integrated Services Network (ISN090105), and the 111 Project (No. B08038).

\section{REFERENCES}

[1] L. Hanzo, Y. Akhtman, L. Wang, and M. Jiang, MIMO-OFDM for LTE, WiFi and WiMAX: Coherent versus Non-coherent and Cooperative Turbo Transceivers. Wiley-IEEE Press, 2010.

[2] T. Pollet, M. Van Bladel, and M. Moeneclaey, "BER sensitivity of OFDM systems to carrier frequency offset and Wiener phase noise," IEEE Trans. Commun., vol. 43, no. 234, pp. 191-193, Feb./Mar./Apr. 1995.

[3] H. Minn, V. Bhargava, and K. Letaief, "A robust timing and frequency synchronization for OFDM systems," IEEE Trans. Wireless Commun., vol. 2, no. 4, pp. 822-839, July 2003.

[4] T. Schmidl and D. Cox, "Robust frequency and timing synchronization for OFDM," IEEE Trans. Commun., vol. 45, no. 12, pp. 1613-1621, Dec. 1997.

[5] C. Chen and J. Li, "Maximum likelihood method for integer frequency offset estimation of OFDM systems," Electron. Lett., vol. 40, no. 13, pp. 813-814, June 2004.

[6] Z. Liu, B. Weng, and Q. Zhu, "Frequency offset estimation for differential OFDM," IEEE Trans. Wireless Commun., vol. 4, no. 4, pp. 1737 - 1748, July 2005.

[7] S. Stein, "Algorithms for ambiguity function processing," IEEE Trans. Acoust., Speech, Signal Process., vol. 29, no. 3, pp. 588-599, June 1981.

[8] J.-C. Guey, "The design and detection of signature sequences in timefrequency selective channel," in Proc. of Personal, Indoor and Mobile Radio Communications, Sept. 2008, pp. 1-5.

[9] K. Liu, T. Kadous, and A. Sayeed, "Orthogonal time-frequency signaling over doubly dispersive channels," IEEE Trans. Inf. Theory, vol. 50, no. 11, pp. 2583-2603, Nov. 2004.

[10] W. Kozek and A. Molisch, "Nonorthogonal pulseshapes for multicarrier communications in doubly dispersive channels," IEEE J. Sel. Areas Commun., vol. 16, no. 8, pp. 1579-1589, Oct. 1998.

[11] M. Hussain, "Principles of high-resolution radar based on nonsinusoidal waves. II. Generalized ambiguity function," IEEE Trans. Electromagn. Compat., vol. 31, no. 4, pp. 369 -375, nov 1989.

[12] M. Jankiraman, Design of Multi-Frequency CW Radars. Scitech Pub Inc, 2007. 